\title{
New Catalytic Asymmetric Strategies to Access Chiral Aldehydes
}

\author{
Clément Mazet ${ }^{\star}$
}

\begin{abstract}
The present article describes the recent catalytic asymmetric methods developed in our group to access enantioenriched chiral aldehydes. We are generally aiming at developing complementary strategies based on either homogeneous organometallic catalysts or amino-catalysts and sometimes on a combination of both. Recent successes comprise a perfectly redox-economical iridium-catalyzed asymmetric isomerization of primary allylic alcohols under very mild conditions and the development of a transition metal-catalyzed anti-Markovnikov asymmetric hydroboration of 1,1-disubstituted olefins. The developments of these two processes along with preliminary mechanistic investigations are presented herein.
\end{abstract}

Keywords: Asymmetric catalysis · Chiral aldehydes · Hydroboration · Iridium · Isomerization

Our long-term research program is centered on the development of new catalytic methods to facilitate access to complex natural products and non-natural analogues that are of importance in biology and medicine (i.e. a target-oriented approach). We place particular emphasis on asymmetric catalysis driven in particular by the study of new reactivity patterns and unconventional reaction mechanisms (i.e. a methodology-driven approach).

Current efforts in the group are directed towards the development of general methods to access enantiopure aldehydes with perfectly controlled $\alpha$ - and/or $\beta$-stereocenters. Such chiral aldehydes are highly prevalent in a variety of biologically active natural products, pharmaceutical agents and fragrances as a key functionality. Moreover, because of their intermediate oxidation state and their dual mode of reactivity (i.e. either as nucleophiles or electrophiles), they are also often encountered as pivotal intermediates in the synthesis of more complex scaffolds.

A major concern in our research program is to elaborate new methods that rely on sustainable catalytic processes. In this context, we have recently developed a perfectly redox-economical iridium-catalyzed asymmetric isomerization of primary allylic alcohols that produces a variety of chi-

${ }^{*}$ Correspondence: Prof. C. Mazet Department of Organic Chemistry University of Geneva

30 quai Ernest Ansermet

$\mathrm{CH}-1211$ Geneva

E-mail: clement.mazet@unige.ch ral aldehydes under very mild conditions. In the present article the milestones of the successful development of this reaction will be presented, along with a more recent extension of this methodology that relies on the sequential use of an organometallic catalyst and an amino-catalyst. Our recent results in the development of a transition metal-catalyzed anti-Markovnikov asymmetric hydroboration of 1,1-disubstituted olefins will also be presented. This complementary method gives access to $\alpha$-chiral aldehydes after sequential oxidation of the chiral boranes to the alcohol and then to the aldehyde oxidation state.

\section{Isomerization of Primary Allylic Alcohols}

When we initiated our studies in this area, the quest for highly selective catalysts for the asymmetric isomerization of primary allylic alcohols (PAA) had stagnated for several years. A survey of the literature revealed that no suitable catalyst had been identified for the non-asymmetric version of this transformation, in particular for PAA with highly substituted double bonds (as it is the case for most prochiral substrates). ${ }^{[1,2]}$ Therefore, we first set out to develop a general method for the non-asymmetric isomerization of PAA that would cover a diverse array of polysubstituted substrates. The identification of a catalytic system that would operate under mild reaction conditions was a major concern in view of the subsequent development of an asymmetric version of this isomerization reaction.

We decided to tackle this problem based on the common observation that isomerizations are the most recurrent competing pathways identified during the hydrogenation of $\mathrm{C}=\mathrm{C}$ double bonds. ${ }^{[3]}$
We hypothesized that by fine-tuning the reaction conditions, it should be possible to deviate hydrogenation catalysts from their initial task in order to promote preferentially the isomerization pathway(s). In the early 1980s, Crabtree and Stork independently demonstrated that complex 1 (i.e. the Crabtree catalyst) catalyzed the directed hydrogenation of a variety of PAA, homoallylic alcohols and secondary allylic alcohols. ${ }^{[4,5]}$ The remarkably mild conditions, the high level of predictability for diastereoselective hydrogenations and the virtual insensitiveness of the metal center with respect to the substitution of the $\mathrm{C}=\mathrm{C}$ double bond of the substrate have favored the implementation of this complex in the main toolbox of the synthetic chemist. In addition, a couple of reports described either undesired isomerizations or even exclusive isomerization for some specific olefinic substrates with catalyst $\mathbf{1}$, thus lending credence to the feasibility of our initial hypothesis. ${ }^{[6,7]}$

Early in our studies, we identified that generating the active form 1- $(\mathrm{H})$, of complex 1 by bubbling molecular hydrogen through a THF solution followed by extrusion of the dissolved molecular hydrogen prior to substrate addition led to the exclusive and quantitative isomerization of PAA into aldehydes. With rigorous application of this new protocol we never observed any traces of the hydrogenation product. Further optimizations showed that - in analogy with hydrogenation reactions - the $\mathrm{BAr}^{\mathrm{F}}$ anion led to increased reaction rates when compared to the less dissociated $\mathrm{PF}_{6}$ anion $\left(\mathrm{BAr}^{\mathrm{F}}=\right.$ tetrakis[3,5-bis-(trifluoromethyl)phenyl]borate). The scope of this new method turned out to be relatively broad and a variety of PAA was isomerized quantitatively in acceptable reaction times (1-24 h), using moderate catalyst loadings $(0.25-5.0 \mathrm{~mol} \%)$ 
and mild experimental conditions (23-65 ${ }^{\circ} \mathrm{C}$ ). Later, systematic investigations on the structure of the achiral catalyst revealed that the specific combination of a trialkylphosphine with a nitrogen-containing ligand was crucial to obtain a catalytically active iridium complex for the isomerization of PAA (Scheme 1). ${ }^{[8]}$

Building on these key observations, we identified ligand scaffold $\mathbf{2}$ as a potential candidate for the development of an asymmetric version of the isomerization of PAA into aldehydes. The original synthesis of this scaffold had been described by Helmchen and co-workers in the early 1990s in the context of the Pd-catalyzed asymmetric allylic alkylation reactions.[9] At that time, only aryl-phosphine derivatives had been reported, presumably for their ease of synthesis and manipulation in standard laboratory atmosphere. Notably, this scaffold has been subsequently used in Rh- and Ir-catalyzed asymmetric hydrogenations by the Imamoto and Pfaltz groups respectively. ${ }^{[10]}$ Keeping our synthetic constraints in mind and following the highly modular synthetic route developed by Helmchen, we rapidly accessed a series of air-sensitive (dialkyl)-phosphino-alkyloxazoline ligands. The corresponding air-stable [(PN) $\operatorname{Ir}(\operatorname{cod})] \cdot$ BAr $^{\mathrm{F}}$ complexes were obtained in good yields (60-88\%) following literature procedures (Scheme 2). ${ }^{[11]}$

Preliminary investigations on a model substrate showed that (i) these complexes displayed a catalytic activity similar to the achiral catalyst $\mathbf{1}$, (ii) the reaction was highly dependent on the size of the P-alkyl substituents; the bulkier substituents leading to the highest enantioselectivities. Under the optimized conditions, the isomerization product was obtained with unprecedented yield and enantioselectivity (98\% yield, $95 \%$ ee or $75 \%$ yield, $97 \%$ ee.$^{[12]}$

The generality of the reaction was subsequently investigated with the most reactive and selective catalysts. The reaction turned out to be relatively substrate-dependent. Whereas $(E)$-configured aryl-alkyl PAA with large alkyl substituents were isomerized with high yields and enantioselectivities, analogous substrates with a small alkyl substituent or a (Z)-configured double bond led to lower yields and enantioselectivities. Interestingly, a competing $(E) /(Z)$ isomerization was observed for the most difficult substrates. Purely alkylsubstituted PAA were revealed to be more challenging to isomerize with high level of enantioselectivity.

Compelling evidence gathered through a series of complementary experiments (X-ray analysis, labeling experiments, ${ }^{1} \mathrm{H}$ NMR identification of reactive intermediates...) led us to propose a plausible mechanism for the isomerization of PAA with these $[(\mathrm{PN}) \operatorname{Ir}(\mathrm{cod})] . \mathrm{BAr}^{\mathrm{F}}$ complexes under

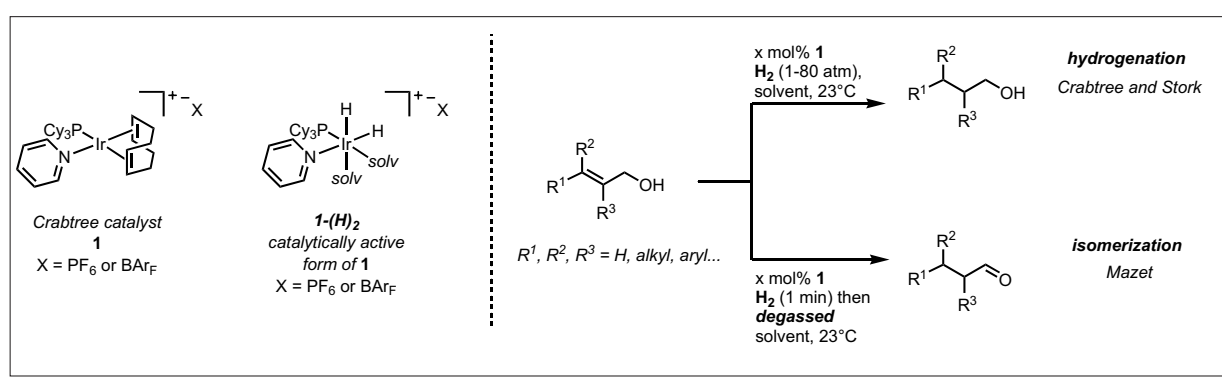

Scheme 1. Crabtree catalyst 1 and its catalytically active dihydride form. Experimental protocols for its use as either a hydrogenation or an isomerization catalyst for PAA.

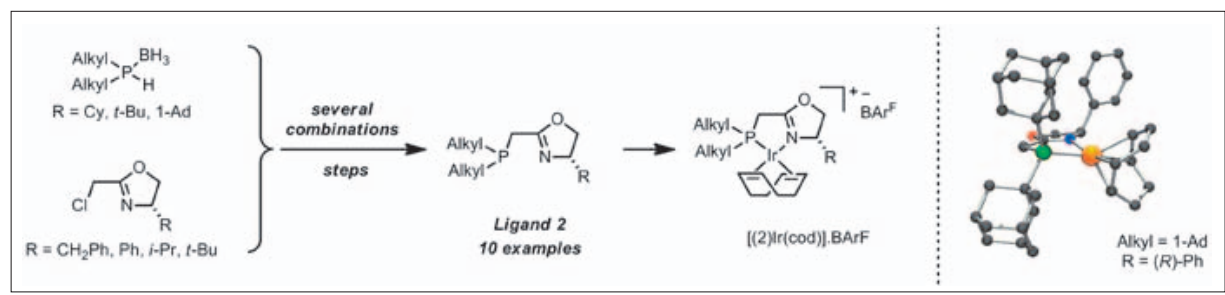

Scheme 2. Schematic representation of the synthetic route to dialkylphosphino-alkyloxazoline ligands $\mathbf{2}$ and the corresponding cationic iridium complexes. X-ray structure of [((1-Ad;Ph)-2) $\operatorname{lr}(\operatorname{cod})]$.BArF.

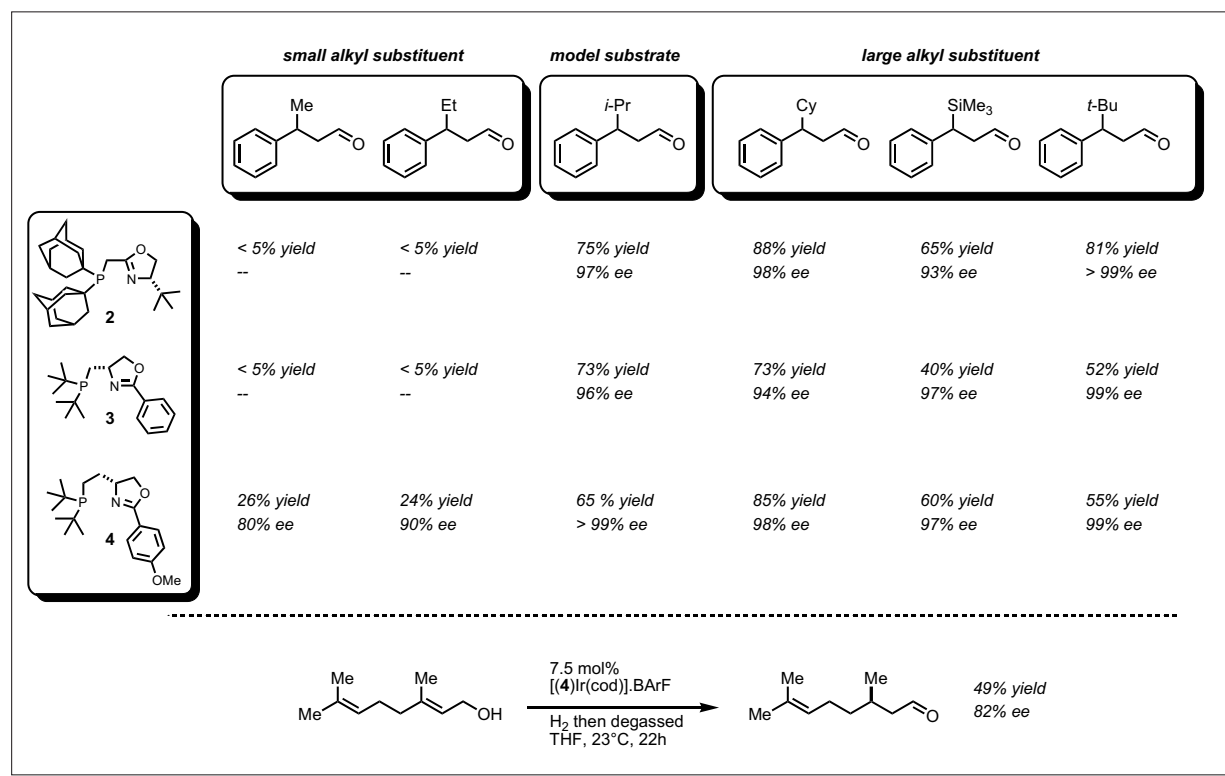

Scheme 3. Comparative results obtained for the asymmetric isomerization of $(E)$-aryl-alkyl substituted PAA with the best ligands of three successive generations of Ir-catalysts.

our experimental protocol. The conformational binding of the PAA on the transient cis-dihydride intermediates was hypothesized to be crucial to explain the high activities and enantioselectivities observed for the more biased substrates and to rationalize the competing $(E) /(Z)$ isomerization pathway. The migratory insertion step was reasoned to be both rate- and enantiodetermining.

On the basis of this mechanism, two other generations of catalysts were synthesized. Elaborated around a readily accessible and less expensive amino-alcohol, the second generation of catalysts $\mathbf{3}$ enabled the isomerization of purely alkyl-containing PAA with unprecedented activity and selectivity levels (ee up to 90\%). ${ }^{[13]}$ Equally accessible, the third generation of catalysts 4 outperform catalysts $\mathbf{2}$ and $\mathbf{3}$ for $(E)$-configured substrates having small alkyl substituents at $\mathrm{C}(3)(e e=80 \%$ for $\mathrm{R}=\mathrm{Me}$ and $e e=90 \%$ for $\mathrm{R}=\mathrm{Et})$. In the case of purely alkyl-containing PAA, the challenging isomerization of geraniol produced citronellal in $49 \%$ yield and $82 \%$ ee (Scheme 3). ${ }^{[14]}$

The identification of enol intermediates during the course of the iridium-catalyzed isomerization reaction prompted us to explore the possibility to generate chiral aldehydes with stereogenic centers on the $\alpha$ - and $\beta$-position using a variety of electrophiles. Since preliminary attempts were met with little success, we decided to follow the recently established HOMO-raising strategy that relies on the use of secondary 
amine-based organocatalysts to introduce the stereogenic center in $\alpha$-position. Our investigations began by establishing the compatibility between the achiral organometallic catalyst $\mathbf{1}$ and several organocatalysts 4-6 ${ }^{[15]}$ using (ethene-1,1-diyldisulfonyl $)$ dibenzene $\left(\left(\mathrm{H}_{2} \mathrm{C}=\mathrm{C}\left(\mathrm{SO}_{2} \mathrm{Ph}\right)_{2}\right)\right.$ as electrophile. After optimization it was found that both catalysts could be used sequentially in the same reaction vessel. During these studies we measured a deviation from the expected 1:1 syn/anti ratio for the most biased substrates. Building on this observation we first developed a kinetic resolution by lowering the stoichiometry in electrophile to 0.5 equivalents. Although the results obtained were not in the useful range, we believe this study will serve as a blueprint for the development of more efficient catalysts for other kinetic resolutions, in particular because related examples in enamine catalysis are scarce in the literature. A more practical advance was achieved when using two chiral catalysts for this sequential transformation. Combining different nucleophiles (PAA) and electrophiles $\left(\mathrm{H}_{2} \mathrm{C}=\mathrm{C}\left(\mathrm{SO}_{2} \mathrm{Ph}\right)_{2}\right.$, NFSI, NCS, DEAD), a variety of $\alpha, \beta$-chiral aldehydes were obtained in acceptable yield, high $d r$ and virtually perfect ee (Scheme 4). Remarkably, even when the mismatch organocatalyst was employed in the second step of the sequence, the corresponding set of diastereomeric products were still obtained with high $d r$ and $e e$ (not shown in Scheme 4). ${ }^{[16]}$

\section{Asymmetric Hydroboration}

During our investigations on alternative modes of activation of our isomerization catalysts we have serendipitously discovered a long-sought anti-Markovnikov hydroboration of 1,1-disubstituted olefins with unprecedented regio- and enantioselectivity levels. Aside from any regioselectivity consideration, this peculiar class of olefinic substrate is notoriously challenging to react with high enantioinduction for mechanistically unrelated mediated or catalyzed asymmetric processes. ${ }^{[17]}$ This has been hypothesized to be due to the difficulty of the chiral auxiliary or the chiral catalyst to distinguish between two geminal substituents. In the specific case of transition metal-catalyzed asymmetric hydroboration reactions, in the very few examples where the anti-Markovnikov adducts were obtained preferentially over the Markovnikov adducts, the yield and enantioselectivity of the borane derivatives were usually low. Surprisingly, the early observation by Marder and Baker that some iridium precatalysts favor the anti-Markovnikov regioselectivity for simple non-prochiral styrenyl derivatives

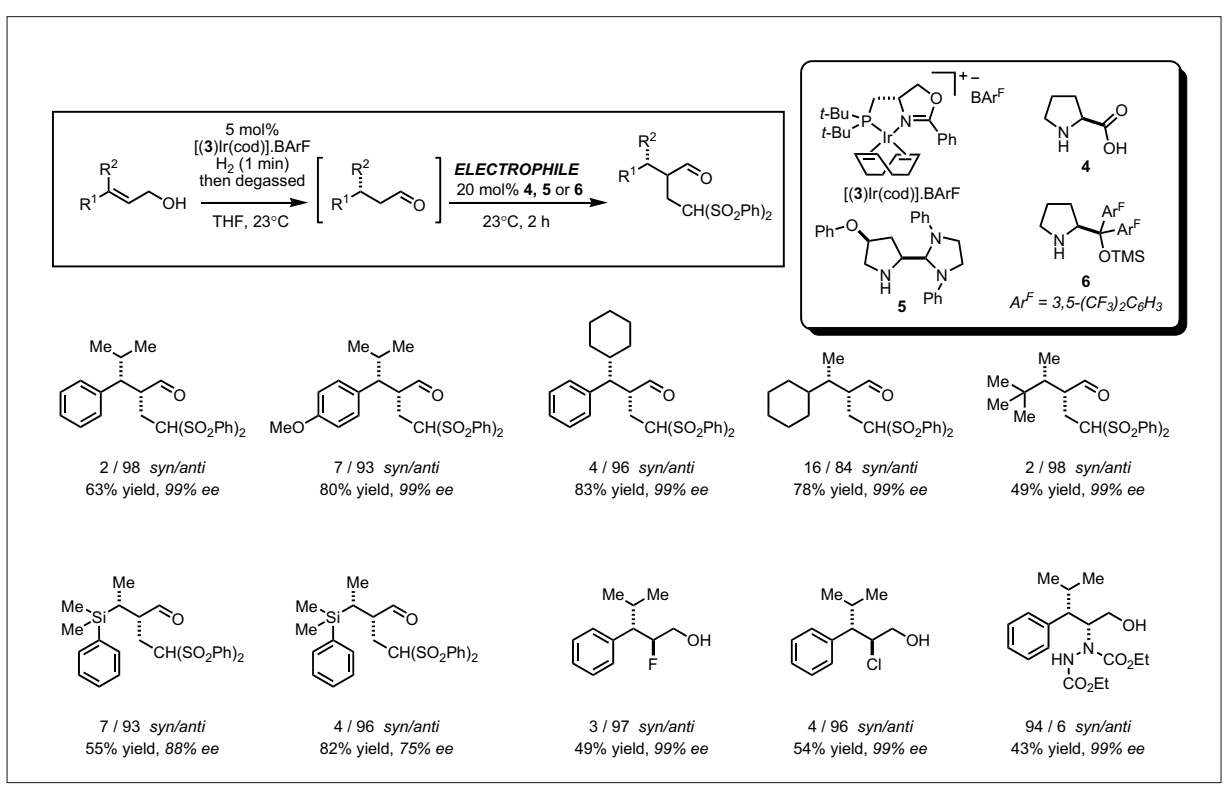

Scheme 4. Scope of $\alpha, \beta$-chiral aldehydes with various combination of nucleophiles (PAA) and electrophiles.

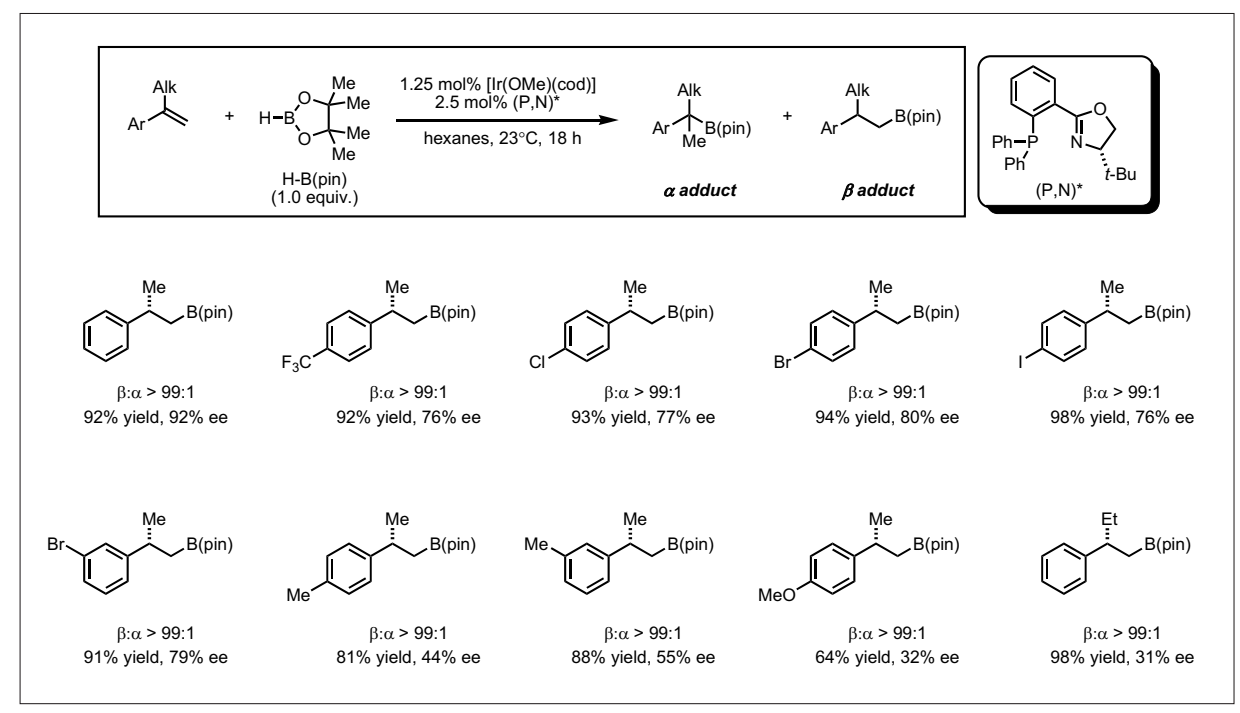

Scheme 5. Scope of the asymmetric hydroboration of 1,1-disubstituted olefins.

had never been exploited in the context of asymmetric catalysis. ${ }^{[18,19]}$ At the outset of our investigations, we established that this trend persisted for $\alpha$-substituted styrenyls when various iridium sources were employed. Consistent with previous studies, the use of pinacolborane as hydroborating agent helped enhance the inherent regiopreference but also led to more stable adducts. Next, a survey of various privileged chiral ligands revealed that - again - the combination of a $\mathrm{P}$ - and $\mathrm{N}$-donor atom was crucial to obtain any measurable enantioinduction. [20]

Under optimized conditions the borane adducts were consistently obtained with perfect anti-Markovnikov regioselectivity and good yields.

The enantioselectivity turned out to be highly dependent on the electronic and steric features of the 1,1-disubstituted olefin employed. Our model substrate, $\alpha$-methylstyrene, yielded the hydroboration adduct with an $e e$ value of $92 \%(\beta: \alpha$ $>99: 1$; $92 \%$ yield). Electron-poor analogues led to $e e$ in the range of $76-80 \%$, whereas electron-rich and bulkier substrates displayed lower selectivity values (31-55\% ee) (Scheme 5).

We next established that all carbon oxidation states - including the aldehyde oxidation state - are accessible from the alkylborane adducts without noticeable epimerization of the $\alpha$-stereogenic center.[21] The 4-bromo-substituted adduct was further derivatized by orthogonal functionalization via sequential Suzuki reactions.[22] First, the halide was selectively engaged in a coupling reaction with phenylboronic acid under benchmark conditions $\left(\mathrm{Pd}(\mathrm{OAc})_{2}\right.$, dppf, $\mathrm{K}_{3} \mathrm{PO}_{4}$ ). Next, the pinacolatoborane moiety was converted into the corresponding trifluoropotassium derivative. Finally, the $\beta$-adducts could be cross-coupled with 


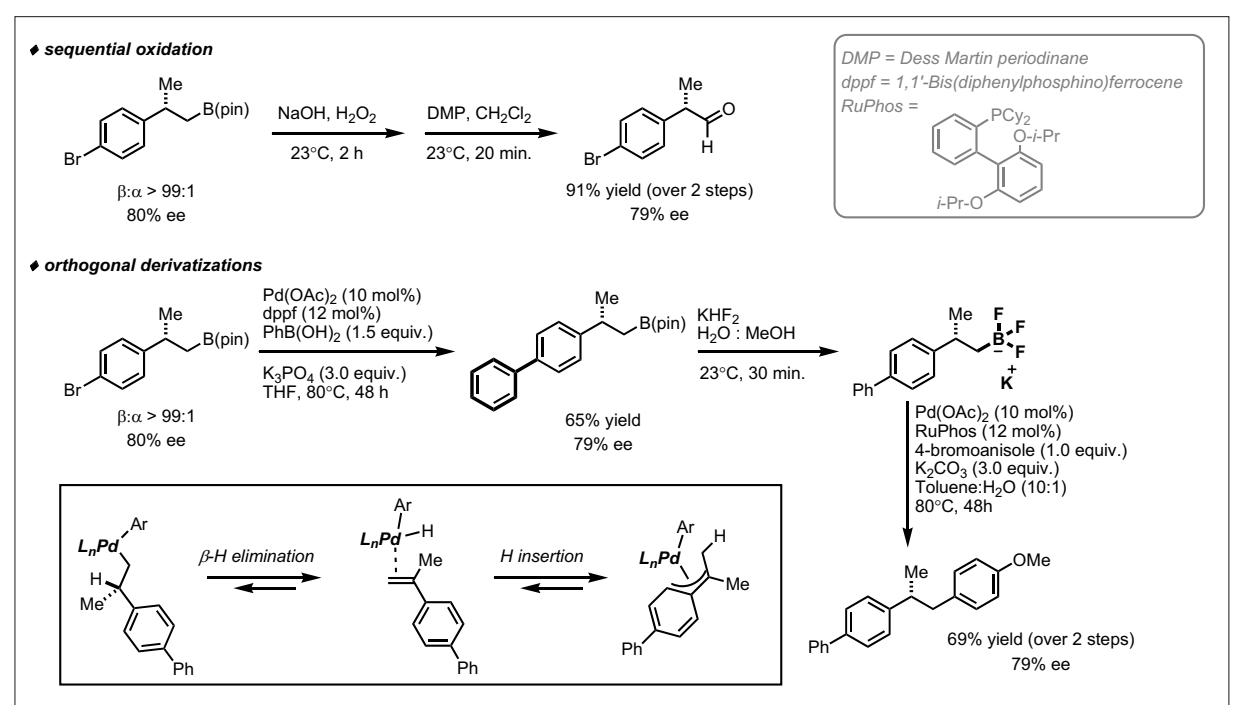

Scheme 6. Derivatization of the pinacolatoborane adducts.

4-bromoanisole without epimerization of the benzylic position applying conditions developed by Molander and co-workers. [23] This demonstrated that under appropriate experimental conditions the $\mathrm{C}\left(\mathrm{sp}^{2}\right)-\mathrm{C}\left(\mathrm{sp}^{3}\right)$ coupling reaction was faster than the concurrent $\beta$-hydride elimination pathway (Scheme 6). [24]

\section{Conclusion}

In the last four years our group has designed two complementary catalytic asymmetric transformations to access chiral aldehydes. Substantial hurdles in the isomerization of PAA have been overcome by detracting a well-established hydrogenation catalyst from its initial task. Identifying the nature of donor atoms one can combine to observe catalytic activity in the isomerization subsequently became instrumental for the development of the asymmetric variant of this reaction. The modification and use of known chiral $(\mathrm{P}, \mathrm{N})$ ligands subsequently enabled access to $\beta$-chiral aldehydes in high yields and unprecedented enantioselectivities. The mechanism of this transformation has been investigated in detail and led to the synthesis of two additional generations of catalysts with improved performances for the most difficult PAA. Access to a variety of $\alpha, \beta$-chiral aldehydes was achieved by combining chiral organometallic iridium catalysts with chiral secondary amine-based organocatalysts. Despite major advances in the asymmetric isomerization of PAA, numerous challenges remain to be addressed regarding the scope of this reaction. In particular, $(Z)$-configured PAA, substrates with either a 2,3-substitution pattern or tetrasubstituted olefins are all resistant to efficient catalytic asymmetric isomerization.

We have also developed an iridiumcatalyzed hydroboration of notoriously challenging 1,1-disubstituted olefins. The corresponding adducts have been obtained with consistently perfect anti-Markovnikov regioselectivity, good yields and promising enantioselectivities for a series of styrenyl derivatives. Although not yet in the practical range, we anticipate that our discovery in the asymmetric hydroboration of 1,1-disubstituted olefins will open up new perspectives for the asymmetric hydrofunctionalization of various classes of alkenes and alkynes. Achieving perfect anti-Markovnikov regioselectivity for hydrofunctionalizations of alkenes was defined as one of the top ten challenges in industrial catalysis ca. 20 years ago. ${ }^{[25]}$ Remarkably, this remains an unsolved chemical problem today.

\section{Acknowledgements}

The research summarized in this article has been carried out by a group of talented and dedicated co-workers to whom I am deeply indebted. Their names appear in the following list of references. Professor A. Alexakis (University of Geneva) is also thanked for a fruitful collaboration. The author also thanks the University of Geneva and the Swiss National Science Foundation, the Société Académique de Genève and Roche for financial support. Johnson-Matthey, Solvias and BASF are also acknowledged for generous gifts of chemicals.

\section{Received: July 15, 2011}

[1] Recent reviews: a) R. C. Van der Drift, E. Bouwman, E. Drent, J. Organomet. Chem. 2002, 650, 1; b) R. Uma, C. Crévisy, R. Grée, Chem. Rev. 2003, 103, 27; c) G. C. Fu, in 'Modern Rhodium-Catalyzed Organic Reactions', Ed. P. A. Evans, Wiley-VCH: Weinheim, 2005, Chap. 4, pp. 79-91; d) V. Cadierno, P. Crochet, J. Gimeno, Synlett 2008, 1105; e) L. Mantilli, C. Mazet, Chem. Lett. 2011, 40, 341 .

[2] a) C. Botteghi, G. Giacomelli, Gazz. Chim. Ital. 1976, 106, 1131; b) K. Tani, T. Yamagata, S. Otsuka, S. Akutagawa, H. Kumobayashi, T. Taketomi, H. Takaya, A. Miyashita, R. Noyori, J. Chem. Soc. Chem. Commun. 1982, 600; c) K. Tani, Pure Appl. Chem. 1985, 57, 1845; d) S. Inoue, H. Takaya, K. Tani, S. Otsuka, T. Sato,
R. Noyori, J. Am. Chem. Soc. 1990, 112, 4897; e) C. Chapuis, M. Barthe, J.-Y. de Saint Laumer, Helv. Chim. Acta 2001, 84, 230; f) S. Bovo, A. Scrivanti, M. Bertoldini, V. Beghetto, U. Matteoli, Synthesis 2008, 2547; g) K. Tanaka, S. Qiao, M. Tobisu, M. M.-C. Lo, G. C. Fu, J. Am. Chem. Soc. 2000, 122, 9870; h) K. Tanaka, G. C. Fu, J. Org. Chem. 2001, 66, 8177; i) F. Boeda, P. Mosset, C. Crévisy, Tetrahedron Lett. 2006, 47, 5021.

[3] R. R. Schrock, J. A. Osborn, J. Am. Chem. Soc 1976, 98, 2134; b) C. S. Chin, J. H. Shin, C. Kim, J. Organomet. Chem. 1988, 356, 381; c) Y. Sun, R. N. Landau, J. Wang, C. LeBlond, D. G. Blackmond, J. Am. Chem. Soc. 1996, 118, 1348.

[4] G. Stork, D. Kahne, J. Am. Chem. Soc. 1983, 105, 1072; b) R. H. Crabtree, M. W. Davis, Organometallics 1983, 2, 681.

[5] R. H. Crabtree, Acc. Chem. Res. 1979, 12, 331.

[6] a) C. Fehr, I. Farris, Angew. Chem. Int. Ed. 2006, 45, 6904; b) M. Krel, J.-Y. Lallemand, C. Guillou, Synlett 2005, 2043; c) D. Solé, X. Urbaneja, J. Bonjoch, Org. Lett. 2005, 7, 5461 d) Y. Kavanagh, C. M. Chaney, J. Muldoon, P. Evans, J. Org. Chem. 2008, 73, 8601

[7] D. Baudry, M. Ephritikhine, H. Felkin, Nouv J. Chim. 1978, 2, 355. In this communication, the authors describe the use of Crabtree catalyst for the isomerization of some PAA but report substantial reproducibility issues. We assume this may be due to either the activation protocol and/or to the use of the less robust original version of the catalyst with the $\mathrm{PF}_{6}$ anion

[8] a) L. Mantilli, C. Mazet, Chimia 2009, 63, 35; b) L. Mantilli, C. Mazet, Tetrahedron Lett. 2009, 50, 4141.

[9] J. Sprinz, G. Helmchen, Tetrahedron Lett. 1993 34, 1769.

[10] a) H. Danjo, M. Higuchi, M. Yada, T. Imamaoto, Tetrahedron Lett. 2004, 45, 603; b) M. G. Schrems, E. Neumann, A. Pfaltz, Angew. Chem. Int. Ed. 2007, 46, 8274.

[11] S. J. Roseblade, A. Pfaltz, Acc. Chem. Res. 2007, 40, 1402.

[12] L. Mantilli, D. Gérard, S. Torche, C. Besnard, C Mazet, Angew. Chem. Int. Ed. 2009, 48, 5143.

[13] L. Mantilli, C. Mazet, Chem. Commun. 2010, $46,445$.

[14] L. Mantilli, D. Gérard, S. Torche, C. Besnard, C. Mazet, Chem. Eur. J. 2010, 16, 12736.

[15] For recent reviews: a) M. Nielsen, C. B Jacobsen, N. Holub, M. W. Paixao, K. A. Jørgensen, Angew. Chem. Int. Ed. 2010, 49, 2668; b) Q. Zhu, Y. Lu, Aust. J. Chem. 2009, 62 951; c) A.-N. R. Alba, X. Companyo, R. Rios, Chem. Soc. Rev. 2010, 39, 2018.

[16] A. Quintard, A. Alexakis, C. Mazet, Angew. Chem. Int. Ed. 2011, 50, 2354.

[17] S. P. Thomas, V. K. Aggarwal, Angew. Chem Int. Ed. 2009, 48, 1896.

[18] S. A. Westcott, T. B. Marder, R. T. Baker, Organometallics 1993, 12, 975 .

[19] a) M. Sato, N. Miyaura, A. Suzuki, Tetrahedron Lett. 1990, 31, 231; b) K. Burgess, M. J. Ohlmeyer, J. Org. Chem. 1988, 53, 5178; c) T. Hayashi, Y, Matsumoto, Y. Ito, Tetrahedron: Asymm. 1991, 2, 601; d) K. Burgess, W. A. van der Donk, M. J. Ohlmeyer, Tetrahedron: Asymm. 1991, 2, 613.

[20] C. Mazet, D. Gérard, Chem. Commun. 2011, 47 , 298.

[21] C. Botuha, M. Haddad, M. Larchevêque, Tetrahedron: Asymm. 1998, 9, 1929.

[22] M. Tobisu, N. Chatani, Angew. Chem. Int. Ed 2009, 48, 3565 .

[23] a) G. A. Molander, L. Jean-Gérard, J. Org. Chem. 2009, 74, 5446; b) R. Martín, S. L. Buchwald, Acc. Chem. Res. 2008, 41, 1461

[24] Y. Iwai, K. M. Gligorich, M. S. Sigman, Angew. Chem. Int. Ed. 2008, 47, 3219.

[25] J. Haggin, Chem. Eng. News 1993, 31, 23. 